\title{
Hospital Length of Stay, Readmissions and Healthcare Costs Through 1 Year for Extremely Preterm Infants With and Without Bronchopulmonary Dysplasia: A Retrospective Glaims Analysis
}

\section{Sujata P. Sarda, ${ }^{1}$ Alexandra Mangili, ${ }^{2}$ Linda Han, ${ }^{3}$ Rajeev Ayyagari, ${ }^{4}$ Wei Gao, ${ }^{4}$ Jessie Wang, ${ }^{4}$ Jing Zhao, ${ }^{4}$ Meredith Mowitz ${ }^{5}$}

'Outcomes Research and Epidemiology (ORE), Shire, Lexington, MA, USA; ${ }^{2}$ Global Development, Shire, Zug Switzerland; ${ }^{3}$ Global Clinical Development, Shire, Lexington, MA, USA; ${ }^{4}$ Analysis Group Inc., Boston, MA, USA ${ }^{5}$ Department of Pediatrics, College of Medicine, University of Florida, Gainesville, FL, USA

\section{BACKGROUND}

- Bronchopulmonary dysplasia (BPD) is the most frequent comorbidity of extremely preterm birth.

- Infants diagnosed with BPD often experience impaired lung function after discharge from the neonatal intensive care unit (NICU). ${ }^{2}$

\section{OBJECTIVE}

To evaluate birth hospital length of stay (LOS), hospita readmissions and healthcare costs for extremely preterm infants ( $\leq 28$ weeks gestational age [GA]), with and without $\mathrm{BPD}$, through 1 year corrected age (CA).

\section{METHODS}

This was a retrospective analysis of the Truven Health MarketScan claims database, which contains health insurance claims and electronic medical records for patients in the United States.

- We included infants born at $\leq 28$ weeks GA who:

- Were admitted to a NICU between January 2009 and June 2016

- Had continuous eligibility through at least 3 months CA Had no congenital heart disease, diaphragmatic hernia or congenital malformations.

- We excluded infants who died before 36 weeks postmenstrual age.

- BPD was identified by International Classification of Diseases, Ninth Revision, Clinical Modification (ICD-9-CM) or ICD-10-CM codes (which included chronic lung disease) during the period from birth to death or the end of continuous eligibility, whichever happened first (severity categorisation for BPD was not available).

- Infants were required to have follow-up for $\geq 3$ months after discharge.

- Outcomes were evaluated utilising a person/time approach due to variable length of follow-up.

- Healthcare costs associated with birth hospitalisation, outpatient visits, emergency room visits and hospital readmissions were evaluated for infants with BPD versus no BPD.

\section{RESULTS}

- Overall, 2805 eligible infants were included in the current analysis (Figure 1)

- 1715 (61.1\%) had BPD, and 1090 (38.9\%) did not have BPD.

- Among infants with BPD, $60.8 \%$ were born at $\leq 26$ weeks GA among infants without BPD, $29.7 \%$ were born at $\leq 26$ weeks GA (Table 1).

- A trend towards higher rates of comorbidities was observed among infants who experienced BPD (Table 1).

- Mean birth hospital LOS was longer for infants with BPD versus those without (Table 2).

- Hospital readmission rates after NICU discharge were significantly higher for infants with BPD versus those without BPD for all causes as well as for respiratory causes specifically (Table 2 ).

- Infants with BPD were more likely to have $>1$ hospital readmission versus infants without $B P D$.

- All healthcare costs were significantly higher for infants with BPD versus those without (Figure 2).

- After birth hospitalisation, outpatient visits accounted for the majority of costs for both groups.
Figure 1. Sample Selection Flowchart of the Truven MarketScan Database

Premature infants $<37$ weeks GA

born in hospital between January 2009 and June 2016 $\mathrm{n}=112,187$

$$
\downarrow
$$

Infants admitted to ICU or NICU within 1 day after birth $\mathrm{n}=\mathbf{4 4 , 7 5 1}$

$$
\downarrow
$$

Infants who died before reaching 36 weeks PMA were excluded $\mathrm{n}=44,212$

$$
\downarrow
$$

Infants diagnosed with congenital heart disease, diaphragmatic hernia or congenital malformations were excluded $n=38,509$

$$
\downarrow
$$

Infants with continuous eligibility from birth to 3-month CA or death, whichever occurred first $\mathrm{n}=\mathbf{2 4 , 0 7 6}$

$$
\downarrow
$$

Infants $\leq 28$ weeks $G A$$$
\mathrm{n}=2805
$$

\begin{tabular}{|c|c|c|}
\hline Characteristic & $\begin{array}{c}\text { Without BPD } \\
n=1090\end{array}$ & $\begin{array}{c}\text { With BPD } \\
n=1715\end{array}$ \\
\hline Female sex, $\mathrm{n}(\%)$ & $528(48.4)$ & $762(44.4)$ \\
\hline \multicolumn{3}{|l|}{ GA, n (\%) $)^{*}$} \\
\hline$<24$ weeks & $18(1.7)$ & $107(6.2)$ \\
\hline 24 weeks & $43(3.9)$ & $268(15.6)$ \\
\hline 25-26 weeks & $263(24.1)$ & $668(39.0)$ \\
\hline 27-28 weeks & $766(70.3)$ & $672(39.2)$ \\
\hline Multiple birth, n (\%) & $264(24.2)$ & $460(26.8)$ \\
\hline \multicolumn{3}{|l|}{ Delivery method, $\mathrm{n}(\%)$} \\
\hline Vaginal & 275 (25.2) & $398(23.2)$ \\
\hline Caesarean & $731(67.1)$ & $1167(68.0)$ \\
\hline Unknown & $84(7.7)$ & $150(8.7)$ \\
\hline \multicolumn{3}{|l|}{ Comorbidities, n (\%) } \\
\hline Chronic respiratory morbidity ${ }^{\dagger}$ & $69(6.3)$ & $448(26.1)$ \\
\hline Intraventricular haemorrhage & $229(21.0)$ & $582(33.9)$ \\
\hline Retinopathy of prematurity & $298(27.3)$ & $743(43.3)$ \\
\hline Patent ductus arteriosus & $383(35.1)$ & $1060(61.8)$ \\
\hline Necrotising enterocolitis & $55(5.0)$ & 209 (12.2) \\
\hline
\end{tabular}

BPD/no BPD cohorts, $\mathrm{n}=\mathbf{2 8 0 5}$

With BPD, $n=1715(61 \%)$

Without BPD, $n=1090(39 \%)$

Table 1. Patient Characteristics During Birth Hospitalisation

\section{CONCLUSIONS}

- BPD places a substantial burden on the healthcare system in the form of longer birth hospital LOS, more hospital readmissions and higher healthcare costs than for infants without BPD.

- Beyond birth hospitalisation, outpatient visits and hospital readmissions are the main drivers of increased healthcare costs among infants with BPD.

\begin{tabular}{|c|c|c|c|}
\hline & $\begin{array}{c}\text { Without BPD } \\
n=1090\end{array}$ & $\begin{array}{c}\text { With BPD } \\
n=1715\end{array}$ & $\begin{array}{l}\text { Adjusted } \\
P \text { V Value* }\end{array}$ \\
\hline \multicolumn{4}{|l|}{ Birth hospitalisation } \\
\hline $\begin{array}{l}\text { Mean } \pm \text { SD length } \\
\text { of stay, days }\end{array}$ & $68 \pm 23$ & $95 \pm 33$ & 0.4308 \\
\hline $\begin{array}{l}\text { Hospital readmission, } \\
\text { all-cause, } \mathrm{n}(\%)\end{array}$ & $131(12.0)$ & $447(26.1)$ & $<0.0001$ \\
\hline $\begin{array}{l}\text { No. of hospitalisations } \\
\text { per person-year } \\
\text { (incidence rate) }\end{array}$ & 0.14 & 0.39 & $<0.0001$ \\
\hline \multicolumn{4}{|l|}{$\begin{array}{l}\text { Length of stay among } \\
\text { infants with } \geq 1 \\
\text { hospital readmission }\end{array}$} \\
\hline Days per person-year & 7.35 & 11.33 & 0.0110 \\
\hline Median, days & 4.00 & 5.00 & - \\
\hline $\begin{array}{l}\text { Infants with >1 } \\
\text { readmission, } \mathrm{n}(\%)\end{array}$ & $20(1.8)$ & $129(7.5)$ & $<0.0001$ \\
\hline $\begin{array}{l}\text { Hospital readmission, } \\
\text { respiratory cause, } n(\%)\end{array}$ & $70(6.4)$ & $290(16.9)$ & $<0.0001$ \\
\hline $\begin{array}{l}\text { No. of hospitalisations } \\
\text { per person-year } \\
\text { (incidence rate) }\end{array}$ & 0.07 & 0.22 & $<0.0001$ \\
\hline \multicolumn{4}{|l|}{$\begin{array}{l}\text { Length of stay among } \\
\text { infants with } \geq 1 \\
\text { hospital readmission }\end{array}$} \\
\hline Days per person-year & 6.05 & 12.10 & $<0.0001$ \\
\hline Median, days & 4.00 & 5.00 & - \\
\hline $\begin{array}{l}\text { Infants with >1 } \\
\text { readmission, n (\%) }\end{array}$ & $8(0.7)$ & $65(3.8)$ & $<0.0001$ \\
\hline
\end{tabular}

REFERENCES
Table 2. Length of Stay During Birth Hospitalisation, and Hospital Readmissions Within 1 Year After Birth Hospitalisation, in Extremely Preterm Infants With or Without BPD
Figure 2. Total Healthcare Costs* per Patient During 1 Yea CA Among Extremely Preterm Infants With or Without BPD When (A) Including Birth Hospitalisation and (B) Excluding Birth Hospitalisation

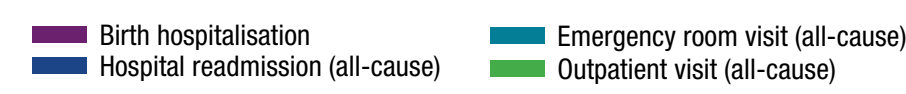

(A) $1,000,000$
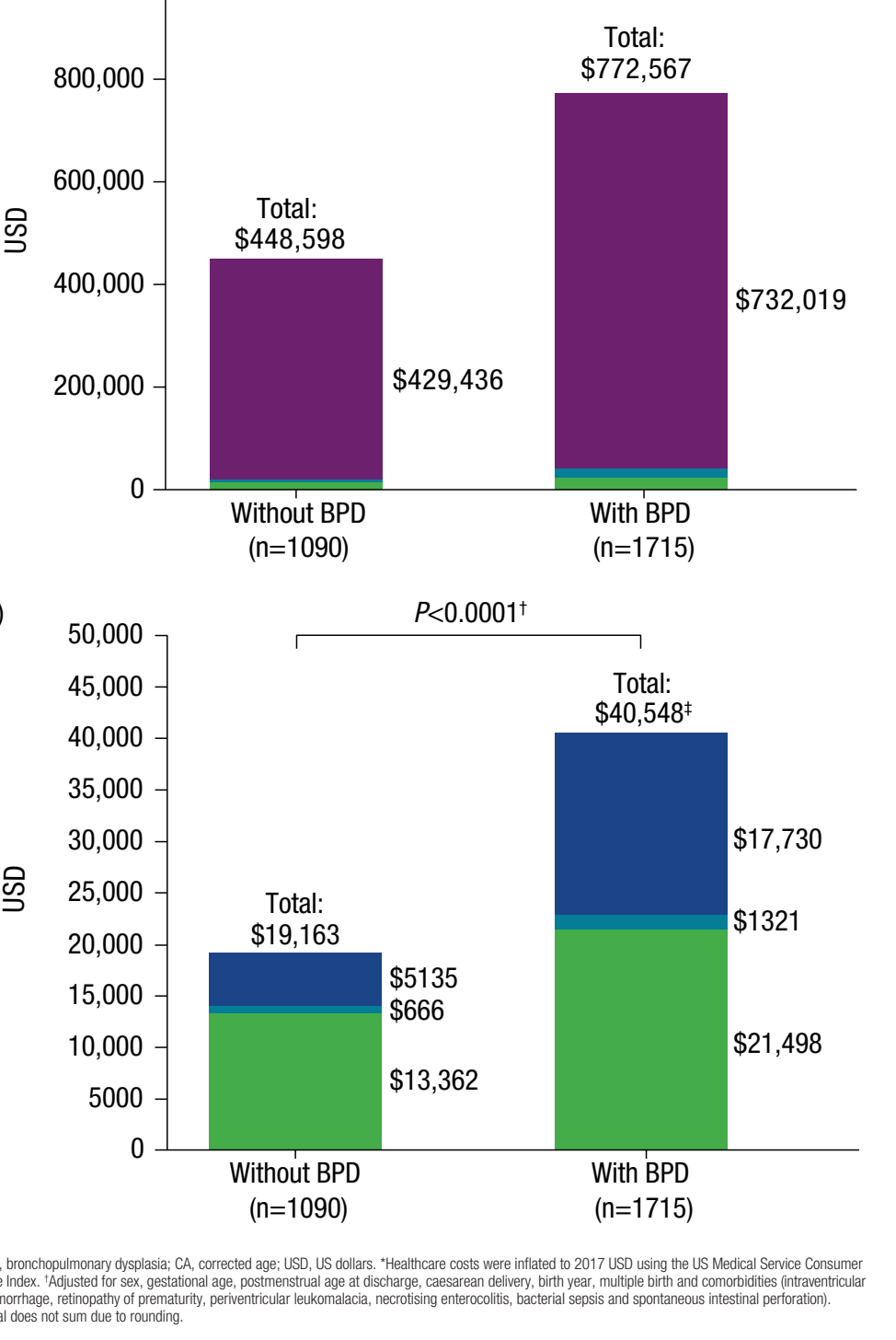